\title{
Adaptación de la unidad de dolor crónico del Complejo Hospitalario de Vigo al estado de alarma por la pandemia SARS-CoV-2
} Adaptation of the chronic pain unit of the Hospital Complex
from Vigo to the state of alarm due to the SARS-CoV-2
pandemic

M. Mayo Moldes, T. Fernández Rodríguez, M. Vieito Amor, M. J. Goberna Iglesias y A. Carregal Raño Unidad de Dolor Crónico. Complejo Hospitalario Universitario de Vigo, Pontevedra, España

\section{ABSTRACT}

COVID-19 infection is responsible for a pandemic. As a result, all outpatient procedures and patient visits to pain management services have been postponed or canceled. This has affected the care of the patient with chronic pain. In our pain management services we have tried to minimize those consequences.

Key words: Telemedicine, COVID, SARS-CoV-2.

\section{RESUMEN}

La infección por COVID-19 es responsable de una pandemia. Como resultado, todos los procedimientos ambulatorios y las visitas de los pacientes a la unidad de dolor han sido pospuestos o cancelados. Esto ha afectado a la atención del paciente con dolor crónico. En nuestra unidad de dolor hemos tratado de minimizar esas consecuencias.

Palabras clave: Telemedicina, COVID-19, SARS-CoV-2.
Durante el estado de alarma por la pandemia SARS-CoV-2, iniciada el 14 marzo de 2020, los hospitales españoles estuvieron saturados, desbordados algunos, ante una situación inesperada, que ha requerido de un gran esfuerzo coordinado para poder asumir la carga de trabajo.

Los pacientes COVID aumentaron exponencialmente, con muy poco tiempo de reacción, en cuestión de días y, para poder atenderlos, muchos servicios hospitalarios modificaron sus circuitos de trabajo.

Las unidades de dolor, mayoritariamente integradas dentro de los servicios de Anestesia, han sido de los servicios que han sufrido gran impacto en esta pandemia.

Mayo Moldes M, Fernández Rodríguez T, Vieito Amor M, Goberna Iglesias MJ, Carregal Raño A. Adaptación de la unidad de dolor crónico del Complejo Hospitalario de Vigo al estado de alarma por la pandemia SARS-CoV-2. Rev Soc Esp Dolor. 2020;27(6):392-393
En este marco excepcional, la Unidad del Dolor Crónico (UDC) del Complejo Hospitalario Universitario de Vigo (CHUVI) intentó optimizar sus recursos, sopesando el riesgo-beneficio para los pacientes: acudir a las consultas para ser atendidos, con el riesgo potencial de contagio, o postergar la asistencia sine die. Tras consensuar las opciones entre el personal de la unidad, y sabiendo que eran cuestiones prioritarias tanto la seguridad del paciente para no contagiarse como su asistencia médica, se decidió suspender las citas presenciales y realizar valoración clínica mediante consulta telefónica, dejando solo para casos prioritarios la asistencia presencial. Nuestra experiencia previa en

Recibido: 11-07-2020

Aceptado: 02-12-2020

Correspondencia: Mónica Mayo Moldes mayomonica@hotmail.com 
teleconsulta con médicos de atención primaria nos facilitó el inicio de esta nueva andadura [1].

En la UDC se realizan anualmente más de 10.000 actos médicos, y el número de pacientes crece exponencialmente cada año. Con este volumen de pacientes, el retraso en las consultas iba a suponer un aumento inasumible de la carga asistencial tras el estado de alarma, sumado al periodo estival, donde las vacaciones del personal obligan a reducir el número de consulta.

Además, el acceso a la UDC está limitado físicamente debido a las características arquitectónicas del edificio.

Esta situación ha supuesto un reto para todos y en la UDC nos ha dado la oportunidad de lograr adaptarnos a situaciones adversas. Nos ha permitido replantearnos algunas cuestiones sobre cuál es la mejor forma de realizar la valoración clínica de nuestros pacientes.

Siguiendo las directrices de los protocolos que sociedades de dolor que se fueron publicando, adaptamos las agendas para la realización de consultas telemáticas (2)-4).

Los cambios realizados en la actividad asistencial fueron los siguientes:

- Todos los pacientes que estaban citados en las consultas durante el estado de alarma fueron valorados por un médico especialista en dolor por vía telefónica. En dicha llamada eran informados del riesgo de contagio por el desplazamiento al hospital, y se les ofertó la posibilidad de asistencia telefónica. En caso de poder resolver la consulta de esta forma, se les valoró clínicamente y se pautó tratamiento con sus recomendaciones, citándolos para una consulta sucesiva en el plazo que consideró el médico responsable.

- Si el paciente, de forma libre y voluntaria, prefirió ser asistido de forma presencial, se les informó de las recomendaciones oficiales y de que se les daría una cita en el plazo considerado pertinente por su médico y la pandemia.

- En caso de ser necesaria la realización de una técnica intervencionista, tras priorización, se les citó de forma presencial para un día y hora concretos, evitando la presencia de más de 4 personas en la sala de espera de la unidad.

- Asimismo, han sido citados de forma presencial, tras valoración inicial siempre telefónica, todas las primeras consultas y revisiones que precisaban de exploración física exhaustiva para tratar de ajustar lo más posible el diagnóstico del paciente, además de las solicitadas como presenciales por los pacientes.

De esta forma se han realizado todas las revisiones y primeras consultas de pacientes, durante el periodo de alarma, sin apenas causar retraso en la atención.
Todos estos cambios permiten disminuir la ansiedad y la preocupación de los pacientes por su enfermedad crónica y observan que el profesional médico está pendiente del desarrollo y la evolución de su dolor. Además se evita la ansiedad que a muchos de ellos le producía la exposición en el hospital.

Con respecto al mismo periodo del año anterior, durante el estado de alarma solo se ha reducido la actividad asistencial un $20 \%$ (1464 actos médicos del 14 de marzo de 2020 al 25 de mayo de 2020 frente a 1960 en el mismo periodo del 2019), datos extraídos de la base de datos de control de gestión del hospital.

Se han atendido presencialmente a todos los pacientes urgentes que precisaban visita médica y no se ha reprogramado ninguna cita automáticamente. Se les realizó técnica intervencionista si así lo precisaban.

Todos los pacientes han sido informados por un médico y, conjuntamente con el paciente, se ha decidido la mejor opción, valorando en todo caso el riesgobeneficio.

Los pacientes, en general, han mostrado estar muy satisfechos con la atención telefónica realizada por el personal médico y, en el caso de citas de revisión, agradecieron evitar acudir. Sería conveniente haber realizado una encuesta de satisfacción.

La actividad de la UDC de nuestra área durante la pandemia SARS-CoV-2 se ha realizado sin suspensiones ni demoras en la atención, ha evitado desplazamientos innecesarios a la consulta presencial, mejorando la organización de la agenda hospitalaria, optimizando los niveles de prioridad y con alto grado de satisfacción de los pacientes.

\section{BIBLIOGRAFÍA}

1. Carregal Rañó A, Mayo Moldes M, Bustabad B. Telemedicina, una nueva herramienta para la gestión del dolor. Resultados de su implementación en una estructura organizativa de gestión integral (EOXI). Rev Soc Esp Dolor. 2020;27(2):97103. DOI: 10.20986/resed.2020.3756/2019.

2. American Society of Regional Anesthesiology. Recommendations on chronic pain practice during the COVID-19 pandemic [Internet]. 2020. Disponible en: https://www.asra.com/ page/2903/recommendations-on-chronic-pain-practiceduring-the-covid-19-pandemic

3. Shanthanna H, Strand NH, D. A. Provenzano DA et all. Caring for patients with pain during the COVID-19 pandemic: consensus recommendations from an international expert panel. Anaesthesia. 2020;75(5):935-44. DOI: 10.1111/ anae.15076.

4. Singleton, Michael N, and Ellen M Soffin. Daring discourse: are we ready to recommend neuraxial anesthesia and peripheral nerve blocks during the COVID-19 pandemic? A pro-con. Reg Anesth Pain Med. 2020;45(10):831-4. DOI: 10.1136/rapm-2020-101653. 